\title{
Escala diagramática para avaliação da severidade da pinta-preta em frutos de mamoeiro
}

\author{
Marcelo Vivas ${ }^{1}$, Carlos Eduardo Pessanha da Silva Terra ${ }^{1}$, Silvaldo Felipe Da Silveira ${ }^{1}$, Renata Venturim Fontes ${ }^{2}$, \\ Messias Gonzaga Pereira²
}

${ }^{1}$ Laboratório de Entomologia e Fitopatologia, e-mail: silvaldo@uenf.br; ${ }^{2}$ Laboratório de Melhoramento Genético Vegetal, Centro de Ciências e Tecnologias Agropecuárias, Universidade Estadual do Norte Fluminense Darcy Ribeiro, CEP 28013-600, Campos dos Goytacazes, RJ, Brasil. Autor para Correspondência: Silvaldo F. da Silveira (silvaldo@uenf.br)

Data de chegada: 27/07/2009. Aceito para publicação em: 21/10/2009.

\section{RESUMO}

Vivas, M.; Terra, C.E.P.S.; Silveira, S.F.; Fontes, R.V.; Pereira, M.G..Escala diagramática para avaliação da severidade de pinta-preta em frutos de mamoeiro. Summa Phytopathologica, v.36, n.2, p.161-163, 2010.

Com o objetivo de padronizar avaliações da severidade da pinta-preta em frutos de mamoeiro, elaborou-se e validou-se uma escala diagramática com os seguintes níveis de severidade: 0,1 ; 0,$3 ; 0,6 ; 1,2 ; 2,5 ; 5,0 ; 10,0$ e $20,0 \%$. Sem a escala, a maioria dos avaliadores superestimou a severidade da doença. Com a escala, os avaliadores obtiveram melhores níveis de acurácia e precisão, com Palavras-chave adicionais: Carica papaya, Asperisporium caricae, erros absolutos concentrando-se na faixa de 5\%, o que é considerado aceitável em trabalhos de quantificação de severidade de doença. O uso da escala proporcionou ainda boa repetibilidade e elevada reprodutibilidade das estimativas. A escala diagramática proposta mostrou-se adequada para avaliação da severidade da pinta-preta em frutos de mamoeiro.

\section{ABSTRACT}

Vivas, M.; Terra, C.E.P.S.; Silveira, S.F.; Fontes, R.V.; Pereira, M.G.. Diagrammatic scale for assessing severity of back-spot in papaya fruit. Summa Phytopathologica, v.36, n.2, p.161-163, 2010.

A diagrammatic scale of black-spot in papaya fruit was elaborated and validate in order to standardize disease severity assessments. The proposed scale showed disease levels of 0.1 ; $0.3 ; 0.6 ; 1.2 ; 2.5 ; 5.0 ; 10.0$ and $20.0 \%$ of fruit superficial area with lesions. Without the scale, most of the evaluators overestimated the disease severity. When the scale was applied, better accuracy and precision levels were obtained, with absolute errors around 5\%, that can be acceptable in this kind of work. The evaluators presented good repeatability and high reproducibility estimations when the scale was used. The diagrammatic scale was suitable for black-spot severity evaluation in papaya fruits.

Keywords: Carica papaya, Asperisporium caricae, assessment, severity.

A pinta-preta, causada por Asperisporium caricae (Speg.) Maubl., é uma das doenças mais comuns na cultura do mamoeiro. Os sintomas dessa doença se manifestam tanto nas folhas quanto nos frutos. Nos frutos ocorrem inicialmente lesões pequenas, circulares e encharcadas, que evoluem para pústulas maiores, marrons a negras e salientes, podendo atingir $5 \mathrm{~mm}$ de diâmetro (6). As lesões nos frutos podem inviabilizar sua comercialização, além de servirem de portas-de-entrada a outros agentes apodrecedores na fase de pós-colheita.

Considerando-se a inexistência de métodos padronizados para quantificação da severidade de pinta-preta em frutos de mamoeiro, este trabalho teve como objetivo desenvolver uma escala diagramática para avaliação da severidade da doença e analisar os níveis de acurácia, precisão e reprodutibilidade das estimativas geradas com sua utilização.

Para elaboração da escala diagramática foram coletados 93 frutos de mamoeiro com diferentes níveis de severidade de pinta-preta. A face externa de cada fruto foi fotografada com câmera digital. A partir das imagens digitalizadas da face externa de cada fruto determinou-se a área total e a área lesionada com auxílio do programa Quant ${ }^{\circledR}(8)$, obtendo-se desta forma a severidade da doença (porcentagem de área lesionada). Baseando-se na lei de Weber-
Fechner de acuidade visual (2), bem como na forma, distribuição e freqüência das lesões, foi confeccionada uma escala diagramática com oito níveis de severidade: 0,$1 ; 0,3 ; 0,6 ; 1,2 ; 2,5 ; 5,0 ; 10,0$ e 20,0\% de área lesionada (Figura 1).

No teste de validação, imagens de 50 frutos com diferentes níveis de severidade foram inseridas aleatoriamente em slides individuais para visualização no Microsoft PowerPoint $2000^{\circledR}$. A severidade foi avaliada por 10 pessoas, a maioria sem experiência na quantificação de doenças em plantas. Inicialmente, a severidade foi estimada sem o auxílio da escala diagramática e, após sete dias, com o auxílio dessa. Visando avaliar a repetibilidade das estimativas com a escala diagramática, sete dias após a primeira avaliação, nova seqüência dos mesmos frutos foi organizada e uma segunda estimativa visual com auxílio da escala foi efetuada pelos mesmos avaliadores.

A partir dos dados de cada avaliador, determinou-se a acurácia e a precisão por meio de regressão linear simples (Microsoft Excel $2000^{\circledR}$ ) entre a severidade real estimada no programa Quant ${ }^{\circledR}$ (variável independente) e a severidade estimada visualmente (variável dependente), sem e com o uso da escala. A precisão das estimativas foi determinada pelo coeficiente de determinação $\left(R^{2}\right)$ da regressão e pela variância dos erros absolutos (diferenças entre o valor real e 


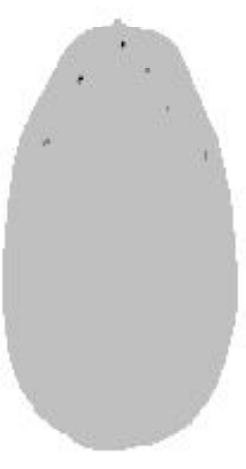

0,1

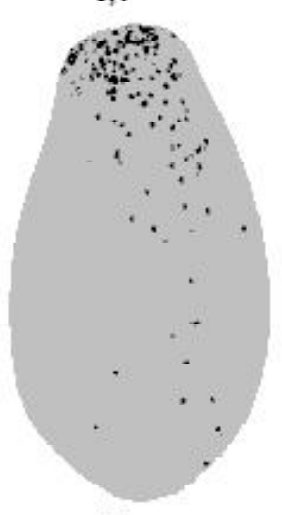

2,5

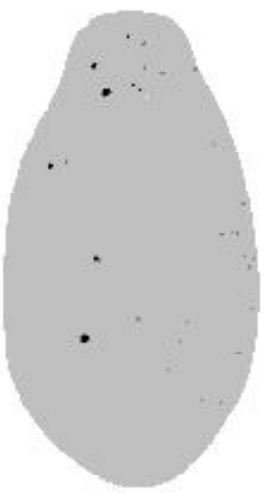

0,3

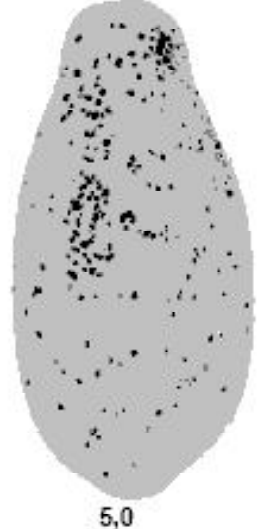

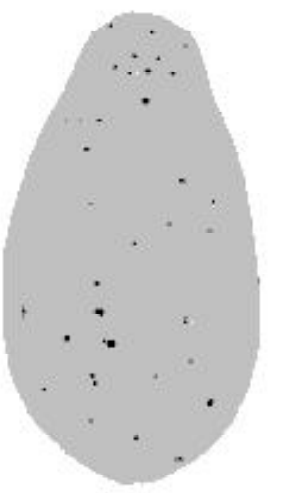

0,6

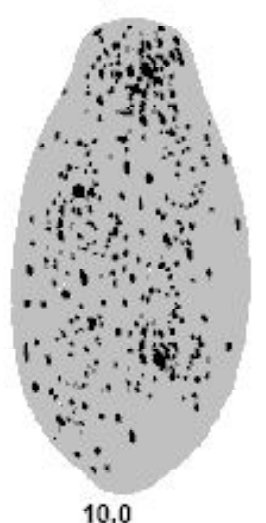

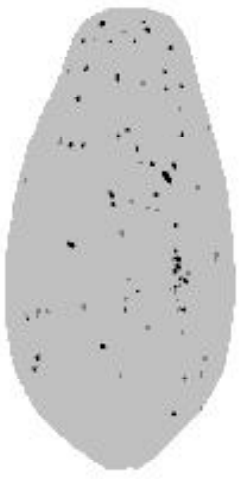

1,2

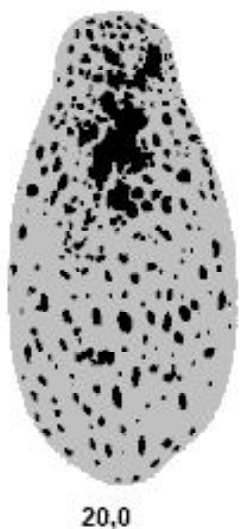

Figura 1. Escala diagramática para avaliação da severidade da pinta-preta em frutos de mamoeiro indicando os níveis de 0,$1 ; 0,3 ; 0,6 ; 1,2 ; 2,5 ; 5,0 ; 10,0$; e $20,0 \%$ de superfície de fruto lesionada (face externa dos frutos em imagem projetada).

Tabela 1. Valor do intercepto $(a)$, coeficiente angular da reta $(b)$ e coeficiente de determinação $\left(R^{2}\right)$ de equações de regressão linear simples relacionando estimativas visuais da severidade de pinta-preta em frutos de mamoeiro, efetuadas por avaliadores, sem e com o auxílio da escala diagramática, com os valores de severidade real, estimada a partir de imagens digitalizadas com auxílio do programa Quant ${ }^{\circledast}$.

\begin{tabular}{|c|c|c|c|c|c|c|c|c|c|}
\hline \multirow{3}{*}{ Avaliador } & \multicolumn{3}{|c|}{ Sem escala } & \multicolumn{6}{|c|}{ Com escala } \\
\hline & \multirow[b]{2}{*}{$\mathbf{a}$} & \multirow[b]{2}{*}{ b } & \multirow[b]{2}{*}{$\mathbf{R}^{2}$} & \multicolumn{3}{|c|}{$1^{\text {a Avaliação }}$} & \multicolumn{3}{|c|}{$2^{\text {a }}$ Avaliação } \\
\hline & & & & $\mathbf{a}$ & b & $\mathbf{R}^{2}$ & $\mathbf{a}$ & b & $\mathbf{R}^{2}$ \\
\hline A & $-0,64$ & $1,83^{*}$ & 0,94 & $-0,45^{*}$ & $0,86^{*}$ & 0,91 & $-0,05$ & $1,11^{*}$ & 0,96 \\
\hline B & $-0,47$ & $2,59^{*}$ & 0,89 & $-0,09$ & 0,96 & 0,93 & $-1,28 *$ & $2,08^{*}$ & 0,91 \\
\hline $\mathrm{E}$ & $6,97 *$ & $4,51 *$ & 0,75 & $-0,71 *$ & $0,86^{*}$ & 0,93 & $-0,62 *$ & $0,86^{*}$ & 0,95 \\
\hline $\mathrm{F}$ & $4,15^{*}$ & $4,17 *$ & 0,78 & $-0,44 *$ & 0,96 & 0,94 & 0,18 & 0,98 & 0,93 \\
\hline G & $7,77 *$ & $4,41 *$ & 0,75 & $-0,62 *$ & $0,77^{*}$ & 0,93 & $-1,08 *$ & $1,16^{*}$ & 0,88 \\
\hline $\mathrm{H}$ & 3,15 & $4,19^{*}$ & 0,80 & $-0,28$ & $0,89^{*}$ & 0,94 & $0,52 *$ & $0,84^{*}$ & 0,90 \\
\hline I & $-0,30$ & $3,95^{*}$ & 0,92 & $-0,10$ & $1,22 *$ & 0,95 & 0,42 & 1,03 & 0,91 \\
\hline
\end{tabular}

*Asterisco indica que a hipótese de nulidade $(a=0$ ou $b=1)$ foi rejeitada pelo teste $t(\mathrm{P}=0,05)$.

estimado). A acurácia foi avaliada por meio dos parâmetros "a" e "b" da equação de regressão, comparando-os, respectivamente, aos valores 0 e 1, pelo teste "t" ( $\mathrm{p}<0,05)$, sem e com uso da escala.

Sem a utilização da escala os valores do intercepto relacionados a $60 \%$ dos avaliadores diferiram significativamente de zero
$(\mathrm{P}=0,05)$, indicando a presença de desvios constantes e de maiores amplitudes (Tabela 1). Com exceção de três avaliadores (A, B e I), os demais superestimaram consistentemente $(\mathrm{P}=0,05)$ a severidade da pinta-preta do mamoeiro, o que foi indicado pela presença de desvios positivos constantes para todos os níveis de severidade da 
doença. Com a utilização da escala, os valores do intercepto de $40 \%$ e $50 \%$ dos avaliadores diferiram significativamente de zero $(\mathrm{P}=0,05)$, na primeira e na segunda avaliação, respectivamente. Todavia, nestas avaliações a grande maioria dos desvios foram negativos e de baixa amplitude, indicando discreta tendência de subestimativa da área lesionada superficial dos frutos.

Com relação aos valores do coeficiente angular da reta, $100 \%$ dos avaliadores apresentaram valores significativamente diferentes de $1(\mathrm{P}=0,05)$, sem a utilização da escala diagramática, indicando a presença de desvios sistemáticos (Tabela 01). Com a utilização da escala, 70\% dos avaliadores na primeira (A, C, E, G, H, I e J) e na segunda (A, B , C , D, E, G e H) avaliações apresentaram coeficientes angulares significativamente diferentes de $1(\mathrm{P}=0,05)$, indicando que a utilização da escala melhorou a acurácia das estimativas visuais (Tabela 1). Quanto à precisão, estimativas visuais da severidade sem a utilização da escala diagramática resultaram em valores de $\mathrm{R}^{2}$ entre 0,75 e 0,96, com média de 0,84 (Tabela 1 ). Com a utilização da escala, na primeira avaliação os valores de $\mathrm{R}^{2}$ foram superiores a 0,87 , com média de 0,93 , enquanto que na segunda avaliação, os valores de $\mathrm{R}^{2}$ foram de 0,88 a 0,97 , com média de 0,92 (Tabela 1 ). Portanto, a precisão das estimativas visuais com auxílio da escala diagramática aumentou, assemelhando-se ao verificado noutros estudos de validação de escalas $(1,4,5,7)$, sendo muito próximos à média de 0,95, valor desejável segundo Kranz (3).

Em geral, os avaliadores tiveram boa repetibilidade nas estimativas, pois a análise de regressão linear entre os dados da segunda avaliação em relação à primeira avaliação com o uso da escala apresentaram valores de $\mathrm{R}^{2}$ entre 0,85 e 0,97, com média de 0,92 (dados não apresentados).

A escala diagramática proposta para avaliação da severidade de pinta-preta em frutos de mamoeiro mostrou ser de fácil utilização, pois auxiliou nas estimativas da doença, com bons níveis de acurácia e precisão. A escala pode ser aplicada a uma ampla gama de situações: levantamentos de campo, estudos de progresso e disseminação da doença, resistência quantitativa de germoplasmas de mamoeiro, bem como em estudos de controle da doença nos frutos em pré e pós-colheita.

\section{AGRADECIMENTOS}

Os autores expressam seus agradecimentos aos colegas que participaram das avaliações de severidade da doença. A Financiadora de Estudos e Projetos (FINEP), a Universidade Estadual do Norte Fluminense Darcy Ribeiro (UENF) e a Caliman Agrícola S/A pelos suportes financeiros e logísticos.

\section{REFERÊNCIAS BIBLIOGRÁFICAS}

1. Díaz, C.G.; Bassanezi, R.B.; Bergamin Filho, A. Desenvolvimento e validação de uma escala diagramática para Xanthomonas axonopodis pv. phaseoli em feijoeiro. Summa Phytopathologica, Jaboticabal, v.27, n.1, p.35-39, 2001.

2. Horsfall, J.G.; Cowling, E.B. Pathometry: the measurement of plant disease. In: Horsfall, J.G.; Cowling, E.B. (Eds.) Plant disease: an advanced treatise - how disease develops in populations. New York: Academic Press, 1978. v.2, p.119-136.

3. Kranz, J. Measuring plant disease. In: Kranz, J.; Rotem, J. (Eds.) Experimental techniques in plant disease epidemiology. Heidelberg: Springer-Verlag, 1988, p.35-50.

4. Leite, R.M.V.B.C.; Amorim, L. Elaboração e validação de escala diagramática para mancha de Alternaria em girassol. Summa Phytopathologica, Botucatu, v.28, n.1, p.14-19, 2002.

5. Nita, M.; Ellis, M.A.; Madden, L.V. Reliability and accuracy of visual estimation of Phomopsis leaf blight of strawberry. Phytopathology, St. Paul, v.93, p.995-1005, 2003.

6. Rezende, J.A.M.; Francelli M.I. Doenças do mamoeiro. In: Kimati, H.; Amorim, L.; Bergamim Filho, A.; Camargo, L.E.A.; Rezende, J.A.M. (Eds). Manual de Fitopatologia: Doenças das plantas cultivadas. São Paulo: Editora Agronômica Ceres, 1997, p.486-496.

7. Tovar-Soto, A.; Hernandez-Martínez, M.; Cristóbal-Alejo, J.; Romero-Hijo, R.; Mora-Aguilera, G. Escala logarítmica diagramática de severidade de la mancha negra (Colletotrichum gloeosporioides Penz.) en Chirimoyo (Annona cherimola Mill). Revista Mexicana de Fitopatologia, Sonora, v.20, n.1, p.103-109, 2002.

8. Vale, F.X.R.; Fernandes Filho, E.I.; Liberato, J.R. QUANT. A software plant disease severity assessment. In: 8th International Congress of Plant Pathology, 2003. New Zealand. Anais Christchurch New Zealand, p.105, 2003. 